\title{
Multiple cancer-related genes are mutated in p53 mutant patches in human skin
}

Amel Albibas ${ }^{1}$, Matthew Rose-Zerilli ${ }^{1}$, Reuben Pengelly ${ }^{1}$, Chester Lai $^{1,2}$, Gabrielle Lockett $^{1}$, Jeffrey Theaker ${ }^{2}$, Sarah Ennis ${ }^{1}$, John Holloway ${ }^{1}$, Eugene Healy ${ }^{1}, 2$ 1 University of Southampton, Southampton, United Kingdom.

2 University Hospital Southampton NHS Foundation Trust, Southampton, United Kingdom.

\section{INTRODUCTION}

- Keratinocyte skin cancer (cutaneous squamous cell carcinoma (cSCC) and basal cell carcinoma) is the most common cancer globally.

- Sun-exposed skin contains numerous p53 immunopositive patches (PIPs, also known as p53 mutant patches) which are potential precursors of keratinocyte skin cancer.

AlM It is known that PIPs contain TP53 gene mutations, but unclear whether these lesions contain mutations of other cancer-related genes.

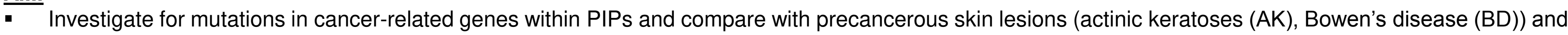
cSCC.

\section{METHODS}

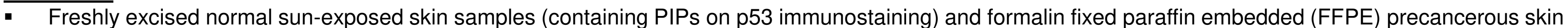
lesions (AKs, BDs) and cSCCs.

- Following p53 immunostaining, PIPs and non-PIPs skin dissected under inverted light microscope.

- Laser capture microdissection used to isolate dysplastic and cancerous areas from FFPE tissue.

- Whole exome sequencing (WES) and targeted sequencing conducted with Illumina HiSeq 2000 platform and MiSeq system respectively.

- $\quad$ Data analysis performed using VarScan 2.3.3.

RESULTS

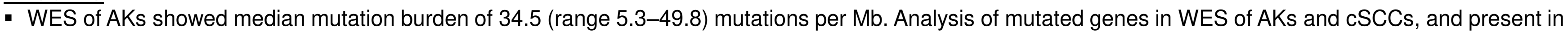
COSMIC (Catalogue of Somatic Mutation in Cancer) database identified 18 genes for subsequent targeted sequencing in larger group of skin lesions (figure 1 ).

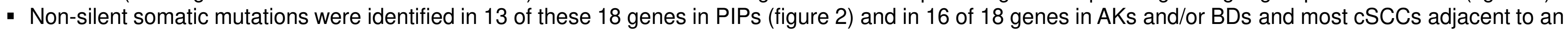
AK or BD (figure 2).

- All 13 genes with non-silent mutations in PIPs were mutated in AKs, BDs and cSCCs in this study with some mutations identical between the different lesions (figure 3).

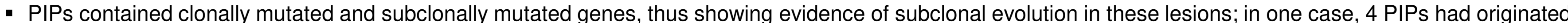
from a shared precursor cell pool and evolved along a common mutation pathway before diverging into separate clones of cells (figure 4).

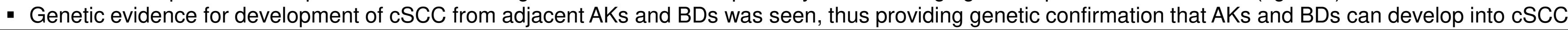

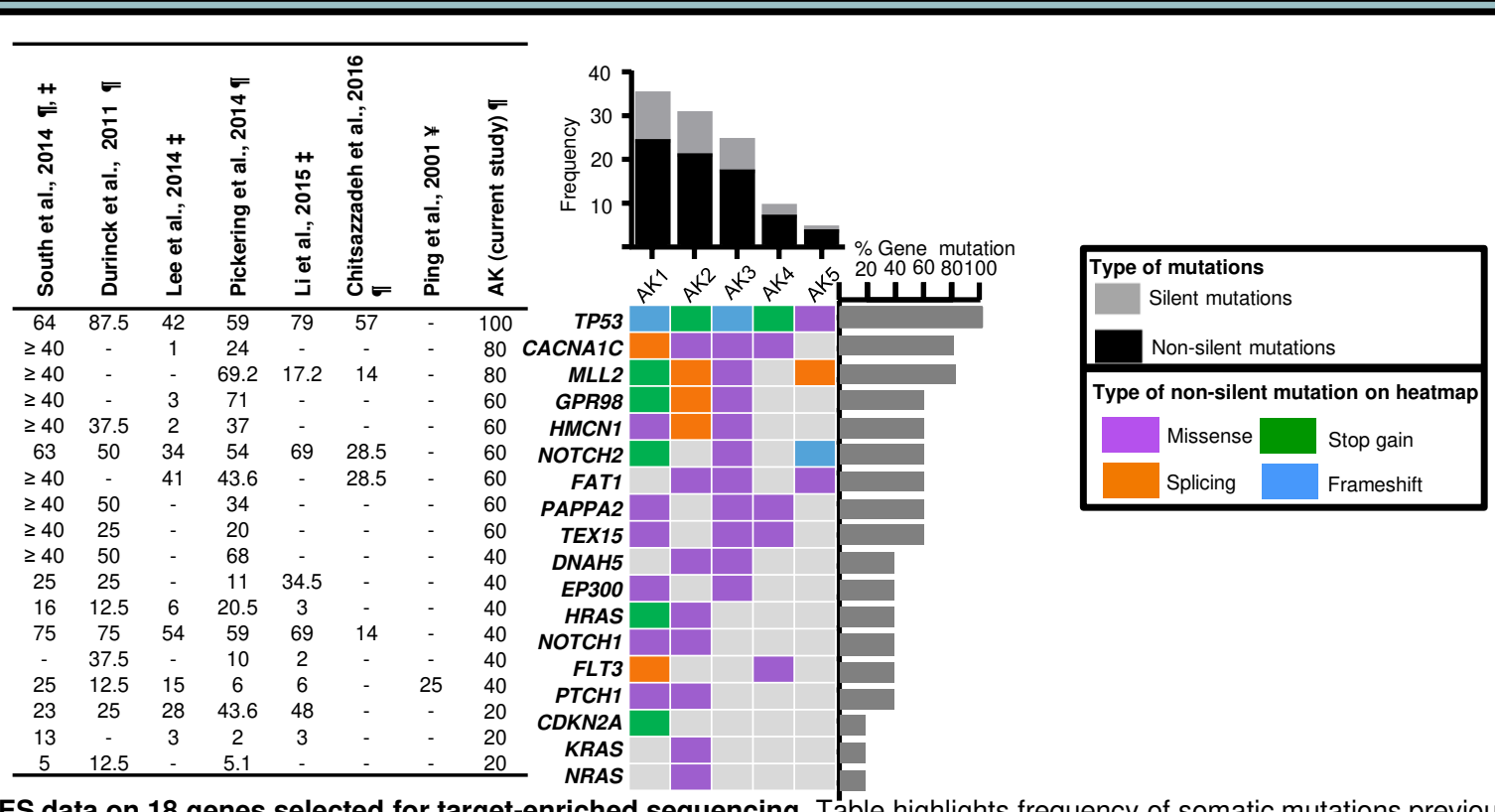

Figure 1: WES data on 18 genes selected for target-enriched sequencing. Table highlights frequency of somatic mutations previously reported within these genes in cSeach
represents mutation frequency of each gene and bar graph above shows frequency of silent and non-silent genetic alterations within dysplastic area of AKs. [-] not reported//not tested, ๆ WES, f targeted sequencing, ¥ Sanger sequencing.
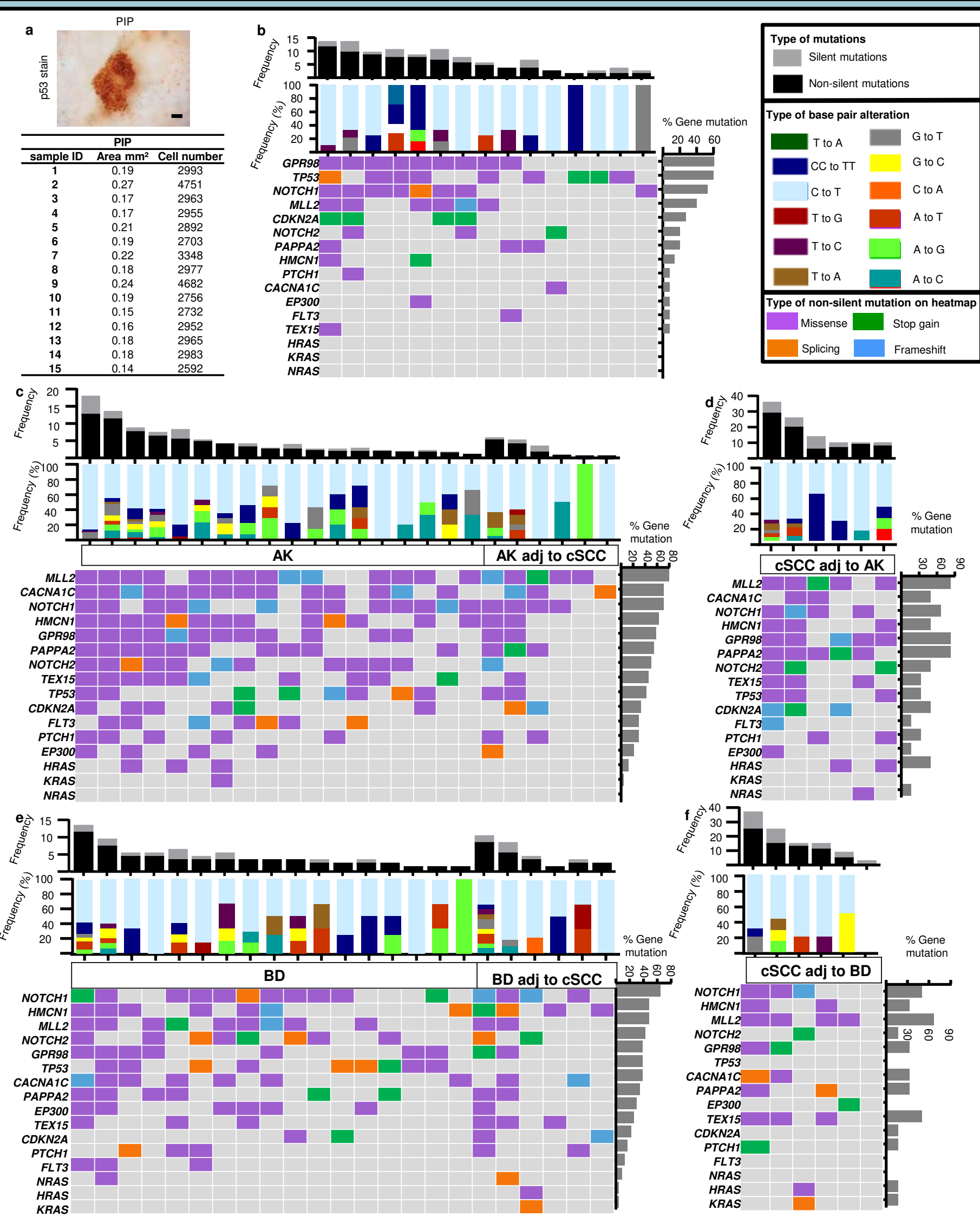
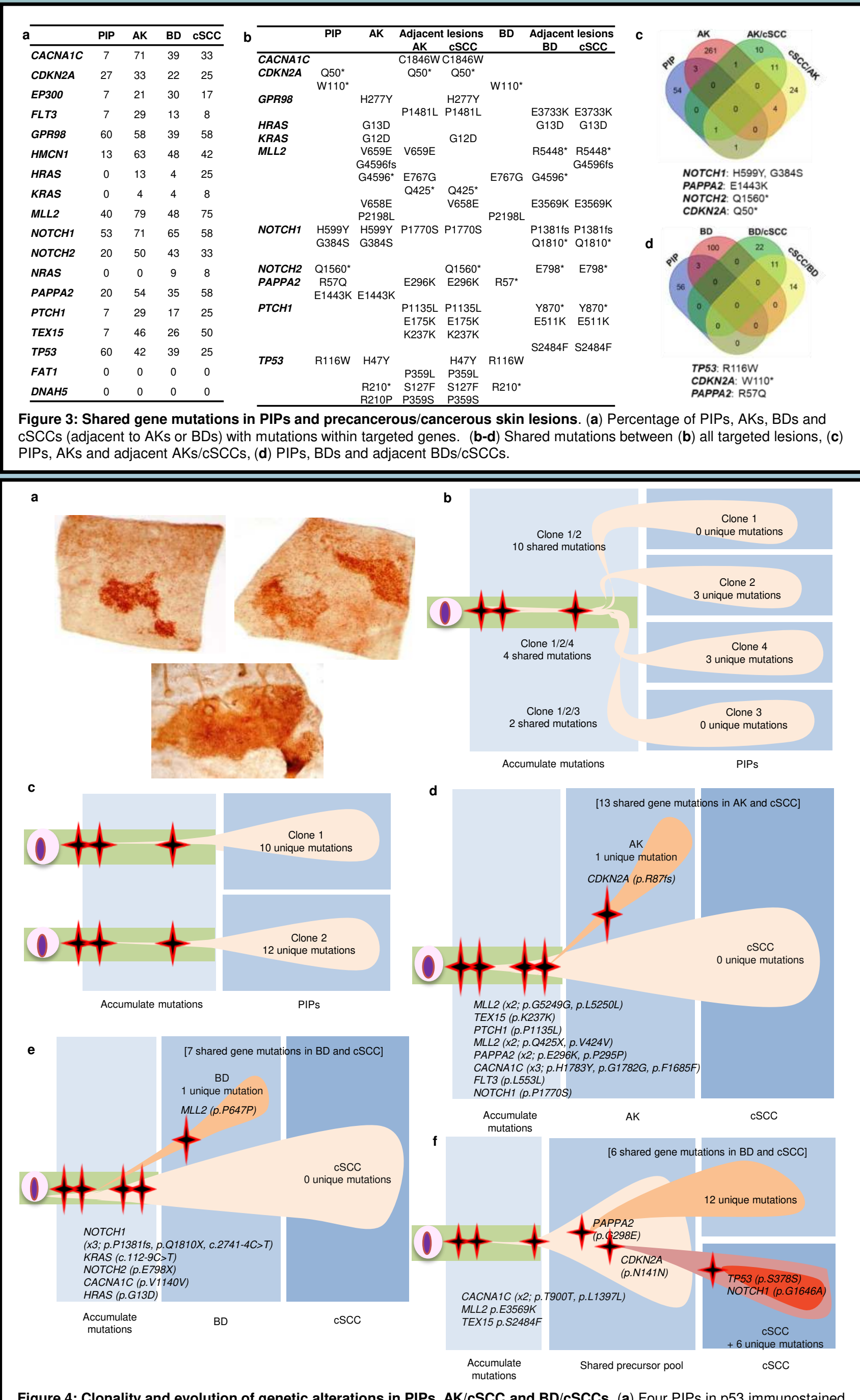

Figure 4: Clonality and evolution of genetic alterations in PIPs, AK/CSCC and BD/cSCCs. (a) Four PIPs in $p 53$ immunostained individual skin sample from another subject. (d-f) Shared and new mutations in adjocent $A$ a

\section{CONCLUSIONS}

p53 immunopostive patches (PIPs) within chronically exposed human skin contain mutations in multiple cancer-related genes, with evidence of subclonal evolution in these lesions.

- Comparison of genetic mutations in cSCCs adjacent to AKs and in cSCCs adjacent to BDs provides genetic confirmation that AKs and BDs can develop into cSCC. 